\title{
THE EQUATIONS OF ELECTRODYNAMICS AND THE NULL INFLUENCE OF THE EARTH'S MOTION ON OF'ICAL AND ELECTRICAL PHENOMENA
}

\author{
By H. R. Hassé.
}

[Received April 12th, 1909.-Read April 22nd, 1909.]

\section{Introduction.}

Tre fundamental equations of the electromagnetic field are usually taken to be those given by Profs. Larmor and Lorentz, deduced in both cases from the hypothesis that matter is formed of discrete electrons. For matter in bulk an averaging process is used in order to obtain the necessary equations. This process introduces new vectors, viz., the electric polarization, the magnetization, and the current of conduction. The scheme of equations is then completed by the assumption of relations connecting these vectors and the two fundamental vectors-electric force and magnetic induction.*

In the following paper the relations connecting these two classes of vectors are deduced from the hypothesis that optical and electrical phenomena are independent of the direction of the motion of the Earth through space. All experiments which have been carried out tend to justify this hypothesis, and their number has been added to recently in an experiment by Prof. Trouton and Mr. Rankine. $t$

The outcome of this hypothesis is that it must be possible to reduce the equations of electrodynamics for moving media to the same form as those of the well known Maxwellian equations for matter at rest. For matter considered as formed of discrete electrons, this was accomplished by Larmor. $\ddagger$

In the case of media in bulk, the special sase of a non-magnetizable dielectric was treated by $\mathrm{H}$. A. Lorentz, $\$$ in 1904, in an investigation as to the possibility of a positive result for the Michelson-Morley experiment arising in the case of propagation through a dielectric.

- Cf. Larmor, Aether and Matter, p. 112.

+ Proc. Roy. Soc., A, Vol. Lxxx, 1907-8, p. 420.

$\ddagger$ Aether and Matter, Chap. xI.

$\$$ Proc. Acad. of Sciences of Amsterdam, 1904, p. $\$ 09$. 
If we distinguish thus between the equations of the electromagnetic field and the equations connecting the subsidiary and the fundamental rectors, which latter we shall refer to as the equations of connection, we shall assume the ordinary equations of the field, but not the equations of connection except in so far as they hold good for matter at rest, the problem being to deduce from the above hypothesis the equations of connection for media in motion.

The investigations contained in this paper were practically completed before the author knew of a paper on the same subject by the late H. Minkowski*; the relation of the work in this paper to that of Minkowski is discussed below.

\section{The Equations of the Electromagnetic Field.}

If we take axes $x, y, z$ fixed in space and a time $t$, the general equations of the electromagnetic field for media in bulk moving with a constant velocity $v$ are, using the vector notation, $\dagger$

$$
\begin{aligned}
\operatorname{curl} H & =\frac{1}{c}\left\{C+\frac{\partial D}{\partial t}+\rho v+c \operatorname{url}[P, v]\right\}, \\
\operatorname{curl} E & =-\frac{1}{c} \frac{\partial B}{\partial t}, \\
\operatorname{div} D & =\rho, \quad \operatorname{div} B=0 .
\end{aligned}
$$

Here $P, C, M$ are the electric polarization, the conduction current, and the magnetization respectively, while $B, E$, and $\rho$ are the magnetic induction, the electric force, and the true electric density; also $H$ and $D$ are defined by

$$
H \equiv B-M, \quad D \equiv E+P .
$$

The units are those used by Lorentz, and $c$ is the velocity of radiation. The operator

the vector

$$
\operatorname{div} A \equiv \frac{\partial}{\partial x} A_{x}+\frac{\partial}{\partial y} A_{y}+\frac{\partial}{\partial z} A_{z},
$$

$$
\text { curl } A \equiv \frac{\partial}{\partial y} A_{z}-\frac{\partial}{\partial z} A_{y}, \frac{\partial}{\partial z} A_{x}-\frac{\partial}{\partial x} A_{z}, \frac{\partial}{\partial x} A_{y}-\frac{\partial}{\partial y} A_{x},
$$

where $A$ is uny vector.

Also, if $A$ and $B$ are two vectors, $[A, B]$ is a vector whose components are $A_{y} B_{z}-B_{y} A_{z}, A_{z} B_{x}-B_{z} A_{x}, A_{x} B_{y}-B_{x} A_{y}$, while the symbol

$$
(A, B) \equiv A_{x} B_{x}+A_{y} B_{x}+A_{*} B_{*}
$$

* Gottinger Nachrichten, 1908.

† For these equations see Larmor, Aether and Matter, Section II, $\$ 73$, or H. A. Lorentz, Ency. der Math. Wiss., V, 14, §33. 
If we refer the equations to axes $x^{\prime}, y^{\prime}, z^{\prime}$ moving with the matter and a time $t^{\prime}(=t)$, we have

$$
x=x^{\prime}+v_{x} t^{\prime}, \quad \ldots,
$$

whence

$$
\frac{\partial}{\partial x^{\prime}}=\frac{\partial}{\partial x}, \quad \ldots,
$$

and

$$
\frac{\partial}{\partial t^{\prime}}=\frac{\partial}{\partial t}+(v \nabla) \equiv \frac{\partial}{\partial t}+v_{x} \frac{\partial}{\partial x}+v_{y} \frac{\partial}{\partial y}+v_{z} \frac{\partial}{\partial z},
$$

and the equations become $\operatorname{curl}^{\prime} H^{\prime}=\frac{1}{c}\left\{C+\frac{\partial D}{\partial t^{\prime}}\right\}$,

$$
\operatorname{curl}^{\prime} E^{\prime}=-\frac{1}{c} \frac{\partial B}{\partial t^{\prime}}
$$

$$
\operatorname{div}^{\prime} D=\rho, \quad \operatorname{div}^{\prime} B=0,
$$

where the new vectors $H^{\prime}$ and $E^{\prime}$ are defined by

$$
H^{\prime} \equiv H-\frac{1}{c}[v, E], \quad E^{\prime} \equiv E+\frac{1}{c}[v, B] .
$$

The operators $\operatorname{div}^{\prime}$ and curl' refer to the moving axes $x^{\prime}, y^{\prime}, z^{\prime}$ just as div and curl referred to the fixed axes.

The problem before us is to consider the possibility of reducing the general equations of the electromagnetic field to a scheme of equations which shall have the same form as the ordinary Maxwellian scheme for matter at rest.

Starting from the equations referred to the moving axes $x^{\prime}, y^{\prime}, z^{\prime}$, let us first apply to them the transformation

$$
t^{\prime \prime}=t^{\prime}-\frac{1}{c^{2}}\left(k, x^{\prime}\right), \quad x^{\prime \prime}=x^{\prime}, \quad y^{\prime \prime}=y^{\prime}, \quad z^{\prime \prime}=z^{\prime},
$$

where $k$ is a constant vector, having components $k_{x}, k_{y}, k_{z}$ as yet undetermined, whence

$$
\frac{\partial}{\partial t^{\prime}}=\frac{\partial}{\partial t^{\prime \prime}}, \quad \frac{\partial}{\partial x^{\prime}}=\frac{\partial}{\partial x^{\prime \prime}}-\frac{k_{x}}{c^{2}} \frac{\partial}{\partial t^{\prime \prime}},
$$

The equations then become

$$
\begin{aligned}
\operatorname{curl} H^{\prime \prime}-\frac{1}{c^{2}}\left[k, \frac{\partial H^{\prime}}{\partial t^{\prime \prime}}\right] & =\frac{1}{c}\left\{\frac{\partial D}{\partial t^{\prime \prime}}+C_{i}^{\prime},\right. \\
\operatorname{curl}^{\prime \prime} E^{\prime}-\frac{1}{c^{2}}\left[k, \frac{\partial E^{\prime}}{\partial t^{\prime \prime}}\right] & =-\frac{1}{c} \frac{\partial B}{\partial t^{\prime \prime}}, \\
\operatorname{div}^{\prime \prime} D-\frac{1}{c^{2}}\left(k, \frac{\partial D}{\partial t^{\prime \prime}}\right) & =\rho, \\
\operatorname{div}^{\prime \prime} B-\frac{1}{c^{2}}\left(k, \frac{\partial B}{\partial t^{\prime \prime}}\right) & =0 .
\end{aligned}
$$


On substituting in the fourth of these equations for $\partial B / \partial t^{\prime \prime}$ from the second, we find

or

$$
\begin{aligned}
\operatorname{div}^{\prime \prime} B+\frac{1}{c}\left(k, \operatorname{curl}^{\prime \prime} E^{\prime}\right) & =0 \\
\operatorname{div}^{\prime \prime}\left\{B-\frac{1}{c}\left[k, E^{\prime}\right]\right\} & =0,
\end{aligned}
$$

whilst equation (2a) may be written

$$
\operatorname{curl}^{\prime \prime} E^{\prime}=-\frac{1}{c} \frac{\partial}{\partial t^{\prime \prime}}\left\{B-\frac{1}{c}\left\lfloor k, E^{\prime}\right]\right\} .
$$

Similarly equation (3a) becomes, on substituting for $\partial D / \partial t^{\prime \prime}$ from (1a),

or

$$
\begin{aligned}
\operatorname{div}^{\prime \prime} D-\frac{1}{c}\left(k, \operatorname{curl}^{\prime \prime} H^{\prime}\right) & =\rho-\frac{(k, C)}{c^{2}} \\
\operatorname{div}^{\prime \prime}\left\{D+\frac{1}{c}\left[k, H^{\prime}\right]\right\} & =\rho-\frac{(k, C)}{c^{2}},
\end{aligned}
$$

whilst equation (1a) can be written

$$
\operatorname{curl}^{\prime \prime} H^{\prime}=\frac{1}{c^{2}}\left\{C+\frac{\partial}{\partial t^{\prime \prime}}\left\{D+\frac{1}{c}\left[k, H^{\prime}\right]\right\}\right\} .
$$

\section{A Special Case of the Transformation.}

In the scheme of equations $(1 b), \ldots,(4 b)$, put

$$
\begin{aligned}
B^{\prime} & =B-\frac{1}{c}\left[k, E^{\prime}\right], \\
D^{\prime} & =D+\frac{1}{c}\left[k, H^{\prime}\right], \\
\rho^{\prime} & =\rho-\frac{1}{c^{2}}(k, C),
\end{aligned}
$$

and the scheme transforms into

$$
\begin{gathered}
\operatorname{curl}^{\prime \prime} H^{\prime}=\frac{1}{c}\left\{C+\frac{\partial D^{\prime}}{\partial t^{\prime \prime}}\right\}, \\
\operatorname{curl} E^{\prime \prime} E^{\prime}=-\frac{1}{c} \frac{\partial B^{\prime}}{\partial t^{\prime \prime}}, \\
\operatorname{div}^{\prime \prime} D^{\prime}=\rho^{\prime}, \quad \operatorname{div}^{\prime \prime} B^{\prime}=0 .
\end{gathered}
$$

As these equations are of the same form as the Maxwellian equations of the electromagnetic field for matter at rest, the problem specitied in $\S 1$ has been solved. In order to complete the scheme of electrodynamical 
equations for matter at rest, we must add to the above equations of the field the equations of connection in the form assumed for matter at rest, viz.,

$$
D^{\prime}=K_{0} E^{\prime}, \quad B^{\prime}=\mu_{0} H^{\prime}, \quad C=\frac{E^{\prime}}{\sigma_{0}} .
$$

Hence, for the original scheme of equations referred to fixed axes, it follows that

$$
\begin{gathered}
D+\frac{1}{c}\left[k, H^{\prime}\right]=K_{0} E^{\prime}, \\
B-\frac{1}{c}\left[k, E^{\prime}\right]=\mu_{0} H^{\prime}, \quad C=\frac{E^{\prime}}{\sigma_{0}} .
\end{gathered}
$$

In the case of a non-magnetizable medium $B=H$, so that the second of these last equations gives us in this case

$$
H-\frac{1}{c}\left[k, E^{\prime}\right]=\mu_{0} H^{\prime}
$$

or

$$
H-\frac{1}{c}[k, E]-\frac{1}{c^{2}}[k,[v, H]]=\mu_{0}\left\{H-\frac{1}{c}[v, E]\right\}
$$

or $\quad H-\frac{1}{c}[k, E]-\frac{1}{c^{2}} v(k, H)+\frac{1}{c^{2}} H(k, v)=\mu_{0}\left\{H-\frac{1}{c}[v, E]\right\}$.

Now let us choose our axes so that the velocity $v$ is parallel to the axis of $x^{\prime}$, i.e., parallel to the axis of $x^{\prime \prime}$, and the above vector equation, written out in full, becomes

$$
\begin{gathered}
H_{x}-\frac{1}{c}\left\{k_{y} E_{z}-k_{z} E_{y}\right\}-\frac{1}{c^{2}} \bar{v}(k, H)+\frac{1}{c^{2}} H_{x} k_{x} \bar{v}=\mu_{0 x} H_{x}, \\
H_{y}-\frac{1}{c}\left\{k_{z} E_{x}-k_{x} E_{z}\right\}+\frac{1}{c^{2}} H_{y} k_{x} \bar{v}=\mu_{0 y} H_{y}+\frac{\mu_{0 y}}{c} \bar{v} E_{z}, \\
H_{z}-\frac{1}{c}\left\{k_{x} E_{y}-k_{y} E_{x}\right\}+\frac{1}{c^{2}} H_{z} k_{x} \bar{v}=\mu_{0 z} H_{z}-\frac{\mu_{0 z}}{c} \bar{v} E_{y},
\end{gathered}
$$

which equations can only be satisfied by putting

$$
k_{y}=k_{z}=0, \mu_{0 x}=1, \quad \mu_{0 y}=\mu_{0 z}=\left(1-\frac{\bar{v}^{2}}{c^{2}}\right)^{-1} \equiv \epsilon, \quad \text { and } k_{x}=\epsilon \bar{v} \text {, }
$$

where $\bar{v}$ is the absolute magnitude of the velocity of the matter, and is no longer a vector.

Similarly, from the equation connecting $D$ and $E^{\prime}$, we should find for the free ether, where $D=E$ and $B=H$, that

$$
K_{0 x}=1, \quad K_{0 y}=K_{0 z}=\epsilon .
$$

As $\epsilon$ involves $\bar{v}$, which ought to disappear from the equations for the free ether, this special case of the transformation is not valid for our purpose. 


\section{The General Transformation.}

The failure of the transformation (A) suggests the possibility that the transformation is not sufficiently general and that it should be extended in a perfectly general manner, the constants of the transformation being afterwards determined so as to satisfy the demands of particular cases.

Starting from the equations $(1 c), \ldots,(4 c)$ of the last paragraph, let us now take the velocity $v$ to be parallel to the axis of $x^{\prime}$, and therefore parallel to the axis of $x^{\prime \prime}$, and put

$$
\left.\begin{array}{rl}
t_{1} & =l t^{\prime \prime} \\
x_{1} & =a_{t} t^{\prime \prime}+a_{x} x^{\prime \prime}+a_{y} y^{\prime \prime}+a_{z} z^{\prime \prime} \\
y_{1} & =b_{t} t^{\prime \prime}+b_{x} x^{\prime \prime}+b_{y} y^{\prime \prime}+b_{z} z^{\prime \prime} \\
z_{1} & =c_{t} t^{\prime \prime}+c_{x} x^{\prime \prime}+c_{y} y^{\prime \prime}+c_{z} z^{\prime \prime}
\end{array}\right\}
$$

where the various coefficients are constants.

Combining the transformations (A) and (B), it is immediately apparent that they give the most general linear transformation from the moving axes $x^{\prime}, y^{\prime}, z^{\prime}$ to the axes $x_{1}, y_{1}, z_{1}$, and from a time $t^{\prime}$ to a time $t_{1}$.

The equations ( $1 c), \ldots,(4 c)$ will then be transformed into equations in which the independent variables of space and time are $x_{1}, y_{1}, z_{1}$, and $t_{1}$ respectively.

For example, the first of the equations (2c) becomes, written out in full,

$$
\begin{aligned}
\left(a_{y} \frac{\partial}{\partial x_{1}}+b_{y} \frac{\partial}{\partial y_{1}}+c_{y} \frac{\partial}{\partial z_{1}}\right) E_{z}^{\prime} & -\left(a_{z} \frac{\partial}{\partial x_{1}}+b_{z} \frac{\partial}{\partial y_{1}}+c_{z} \frac{\partial}{\partial z_{1}}\right) E_{y}^{\prime} \\
+ & \frac{1}{c}\left(l \frac{\partial}{\partial t_{1}}+a_{t} \frac{\partial}{\partial x_{1}}+b_{t} \frac{\partial}{\partial y_{1}}+c_{t} \frac{\partial}{\partial z_{1}}\right) B_{x}^{\prime}=0 .
\end{aligned}
$$

If we therefore put

$$
\begin{aligned}
b_{y} E_{z}^{\prime}-b_{z} E_{y}^{\prime}+\frac{b_{t}}{c} B_{x}^{\prime} & \equiv E_{1 z} \nu_{x} \\
c_{y} E_{z}^{\prime}-c_{z} E_{y}^{\prime}+\frac{c_{l}}{c} B_{x}^{\prime} & \equiv-E_{1 y} \nu_{x} \\
\frac{l}{c} B_{x}^{\prime} & \equiv \frac{B_{1 x}}{c} \nu_{x} \\
a_{y} E_{z}^{\prime}-a_{z} E_{y}^{\prime}+\frac{a_{l}}{c} B_{x}^{\prime} & \equiv 0
\end{aligned}
$$


the above equation transforms into

$$
\frac{\partial}{\partial y_{1}} E_{1 z}-\frac{\partial}{\partial z_{1}} E_{1 y}=-\frac{1}{c} \frac{\partial B_{1, x}}{\partial t_{1}},
$$

which is of the form required for matter at rest.

Similarly the other two equations of the vector equation $(2 c)$ can be brought into the proper form for matter at rest by substitutions of the above type.

These substitutions, twelve in number, can be written in the abbreviated forms, using the vector notation where possible,

$$
\left.\begin{array}{rl}
l B^{\prime} & =\left\{\nu_{x}, \nu_{y}, \nu_{z}\right\} B_{1} \\
{\left[a, E^{\prime}\right]+\frac{a_{t}}{c} B^{\prime}} & =\left\{0,-\nu_{y} E_{1 z}, \nu_{z} E_{1 y}\right\} \\
{\left[b, E^{\prime}\right]+\frac{b_{t}}{c} B^{\prime}} & =\left\{\nu_{x} E_{1 z}, 0,-\nu_{z} E_{1, y}\right\} \\
{\left[c, E^{\prime}\right]+\frac{c_{t}}{c} B^{\prime}} & =\left\{-\nu_{x} E_{1 y}, \nu_{y} E_{1 x}, 0\right\}
\end{array}\right\} .
$$

In un exactly similar way the vector equation (1c) will tiansform into the vector equation

$$
\operatorname{curl}_{1} H_{1}=\frac{1}{c}\left\{C_{1}+\frac{\partial D_{1}}{\partial t_{1}}\right\}
$$

by means of the substitutions

$$
\left.\begin{array}{rl}
l D^{\prime} & =\left\{\lambda_{x}, \lambda_{y}, \lambda_{z}\right\} D_{1} \\
{\left[a, H^{\prime}\right]+\frac{a_{t}}{c} D^{\prime}} & =\left\{0,-\lambda_{y} H_{1 z}, \lambda_{z} H_{1 y}\right\} \\
{\left[b, H^{\prime}\right]+\frac{b_{t}}{c} D^{\prime}} & =\left\{\lambda_{x} H_{1 z}, 0,-\lambda_{z} H_{1 x}\right\} \\
{\left[c, H^{\prime}\right]+\frac{c_{t}}{c} D^{\prime}} & =\left\{-\lambda_{x} H_{1 y}, \lambda_{y} H_{1 x}, 0\right\} \\
C_{1} & =\left\{\frac{1}{\lambda_{x}}, \frac{1}{\lambda_{y}}, \frac{1}{\lambda_{z}}\right\} C
\end{array}\right\} .
$$

and

As a preliminary to determining the constants of the transformation, we notice that equations (5) and (6) give two values for each of the components of $E_{1}$ and $H_{1}$, which must necessarily ugree with one another.

For example, taking $H_{1 z}$, we have, from (6),

$$
H_{1 z}=-\frac{1}{\lambda_{y}}\left(a_{z} H_{x}^{\prime}-a_{x} H_{z}^{\prime}+\frac{a_{t}}{c} D_{y}^{\prime}\right),
$$

and also

$$
=\frac{1}{\lambda_{x}}\left(b_{y} H_{z}^{\prime}-b_{z} H_{y}^{\prime}+\frac{b_{t}}{c} D_{x}^{\prime}\right) \text {, }
$$


which can only agree if

$$
a_{t}=b_{t}=a_{z}=b_{z}=0 \text { and } a_{x} \lambda_{x}=b_{y} \lambda_{y} .
$$

Similarly, taking $H_{1 y}$, we should find that

$$
c_{t}=a_{t}=a_{y}=c_{y}=0 \quad \text { and } \lambda_{z} c_{z}=a_{x} \lambda_{x} ;
$$

and, taking $H_{1 x}$,

$$
c_{t}=b_{t}=b_{x}=c_{x}=0 \text {, }
$$

and

$$
\lambda_{y} b_{y}=\lambda_{z} c_{z} \text {. }
$$

We therefore immediately find that

$$
\begin{gathered}
a_{t}=b_{t}=c_{t}=0, \\
\text { and } \quad b_{x}=c_{x}=a_{y}=c_{y}=a_{z}=b_{z}=0, \\
\lambda_{x} a_{x}=\lambda_{y} b_{y}=\lambda_{z} c_{z}(\equiv \xi) .
\end{gathered}
$$

The equations (5) and (6) only differ as regards the constants by the substitution of $\nu$ for $\lambda$, so that we obtain exactly similar results from a consideration of the different values of $E_{1}$, save that

$$
\nu_{x} a_{x}=\nu_{y} b_{y}=\nu_{z} c_{z}(\equiv \eta \xi),
$$

so that

$$
\frac{\lambda_{x}}{\nu_{x}}=\frac{\lambda_{y}}{\nu_{y}}=\frac{\lambda_{z}}{\nu_{z}}=\frac{1}{\eta} \text {. }
$$

Equations (5) and (6) therefore take the simpler forms

$$
\left.\begin{array}{rl}
l B^{\prime} & =\eta B_{1}\left\{\lambda_{x}, \lambda_{y}, \lambda_{z}\right\} \\
l D^{\prime} & =D_{1}\left\{\lambda_{x}, \lambda_{y}, \lambda_{z}\right\} \\
\xi\left\{\lambda_{x}, \lambda_{y}, \lambda_{z}\right\} H^{\prime} & =H_{1} \lambda_{x} \lambda_{y} \lambda_{z} \\
\xi\left\{\lambda_{x}, \lambda_{y}, \lambda_{z}\right\} E^{\prime} & =E_{1} \eta^{2} \lambda_{x} \lambda_{y} \lambda_{z}
\end{array}\right\} .
$$

Further, equations $(3 c)$ and $(4 c)$ now transform into

$$
\operatorname{div}_{1} D_{1}=\rho_{1}, \quad \operatorname{div}_{1} B_{1}=0,
$$

where

$$
\rho_{1}=\frac{l \rho^{\prime}}{\xi} \text {. }
$$

To complete the scheme of electrodynamical equations for matter at rest, we must add to the above equations of the field the equations of connection in the form

$$
D_{1}=K_{0} E_{1}, \quad B_{1}=\mu_{0} H_{1}, \quad C_{1}=\frac{E_{1}}{\sigma_{0}},
$$

and it now only remains to find the nine constants $\lambda_{x}, \lambda_{y}, \lambda_{i}, \eta, \xi, l$, 
$k_{x}, k_{y}, k_{z}$, the last three of which enter into the definitions of $B^{\prime}, D^{\prime}$, and $\rho$ at the beginning of $\$ 2$.

4. The Determination of the Constants of Transformation.

In the special case of no conduction current, we must have

$$
\iiint \operatorname{div}_{1} D_{1} d x_{1} d y_{1} d z_{1}=\iiint \operatorname{div}^{\prime} D d x^{\prime} d y^{\prime} d z^{\prime}
$$

since motion of a material medium cannot alter the charge per unit volume.

This gives $\quad \xi^{2} l=\lambda_{x} \lambda_{y} \lambda_{z}$,

by using equation $(3 d)$, and the original equation

$$
\operatorname{div}^{\prime} D=\rho .
$$

From the equation

$$
D_{1}=K_{0} E_{1}
$$

we deduce, by means of the equation (7),

$$
D^{\prime}=K_{0} \frac{\xi}{l^{2} \lambda_{x} \lambda_{y} \lambda_{z}}\left\{\lambda_{x}^{2}, \lambda_{y}^{2}, \lambda_{z}^{2}\right\} E^{\prime}
$$

Since

$$
D^{\prime}=D+\frac{1}{c}\left[k, H^{\prime}\right]
$$

and

$$
E^{\prime}=E+\frac{1}{c}[v, B]
$$

this gives us the relation connecting the electric displacement $D$ and the vectors $E, H^{\prime}$ and $B$.

The above equation (8) must hold good for the free ether, i.e., when $D=E$, and $B=H$. We must therefore have

$$
E+\frac{1}{c}\left[k, H^{\prime}\right]=\frac{K_{0} \xi}{\ln ^{2} \lambda_{x} \lambda_{y} \lambda_{z}}\left\{\lambda_{x}^{2}, \lambda_{y}^{2}, \lambda_{z}^{2}\right\} E^{\prime}
$$

Now

$$
H^{\prime}=H-\frac{1}{c}[v, E]
$$

thus $E+\frac{1}{c}[k, H]-\frac{1}{c^{2}}[k,[v, E]]$

$$
=\frac{K_{0} \dot{\xi}}{l^{2} \lambda_{x} \lambda_{y} \lambda_{z}}\left\{\lambda_{x}^{2}, \lambda_{y}^{2}, \lambda_{z}^{2}\right\}\left\{E+\frac{1}{c}[v, H]\right\},
$$


or $\quad E+\frac{1}{c}[k, H]-\frac{1}{c^{2}} v(k, E)+\frac{E}{c^{2}}(k, v)$

$$
=\frac{K_{0} \xi}{l^{2} \lambda_{x} \lambda_{y} \lambda_{z}}\left\{\lambda_{x}^{2}, \lambda_{y}^{2}, \lambda_{z}^{2}\right\}\left\{E+\frac{1}{c}[v, H]\right\} .
$$

Writing out this vector equation in full, we have

$$
\begin{aligned}
E_{x}+\frac{1}{c}\left\{k_{y} H_{z}-k_{z} H_{y}\right\} & =\frac{K_{0} \xi}{l^{2} \lambda_{x} \lambda_{y} \lambda_{z}} \lambda_{z}^{2} E_{x}, \\
E_{y}\left\{1+\frac{k_{x} \bar{v}}{c^{2}}\right\}+\frac{1}{c}\left\{k_{z} H_{x}-k_{x} H_{z}\right\} & =\frac{K_{0} \xi}{l^{2} \lambda_{x} \lambda_{y} \lambda_{z}} \lambda_{y}^{*}\left\{E_{y}-\frac{\bar{v}}{c} H_{z}\right\}, \\
E_{z}\left\{1+\frac{k_{x} \bar{v}}{c^{2}}\right\}+\frac{1}{c}\left\{k_{x} H_{y}-k_{y} H_{x}\right\} & =\frac{K_{0} \xi}{l^{2} \lambda_{x} \lambda_{y} \lambda_{z}} \lambda_{z}^{z}\left\{E_{z}+\frac{\bar{v}}{c} H_{y}\right\} .
\end{aligned}
$$

We must therefore have

$$
\begin{aligned}
& k_{y}=k_{z}=0, \quad K_{0} \xi \lambda_{x}^{z}=l^{2} \lambda_{x} \lambda_{y} \lambda_{:}, \\
& \left.1+\frac{k_{x} \bar{v}}{c^{2}}\right\}^{\prime}=\frac{K_{0} \xi \lambda_{y}^{2}}{l^{2} \lambda_{x} \lambda_{y} \lambda_{z}}=\frac{K_{0} \xi \lambda_{z}^{z}}{l^{2} \lambda_{x} \lambda_{l !} \lambda_{z}},
\end{aligned}
$$

and

$$
k_{x}=\frac{K_{0} \hat{\xi}}{l \eta^{2} \lambda_{x} \lambda_{y} \lambda_{z}} \lambda_{y}^{2} \bar{v}=\frac{K_{0} \xi}{l \eta^{2} \lambda_{x} \lambda_{y} \lambda_{z}} \lambda_{z}^{2} \bar{v},
$$

which can be satisfied by putting $K_{0}=1$,

$$
\begin{aligned}
k_{x}=\frac{\xi}{l \eta^{2}} \frac{\bar{v}}{\lambda_{x}} & =\bar{v}\left(1-\frac{\bar{v}^{2}}{c^{2}}\right)^{-1}=\bar{v} \epsilon, \\
\lambda_{y} & =\lambda_{z}=\lambda_{x} \epsilon^{\underline{k}} .
\end{aligned}
$$

Thus the constant $K_{0}$ for the free ether is unity, which is consistent with the original equations of the electromagnetic field from which we set out, which are based on the hypothesis of a stagnant ether.

From the equation

$$
B_{1}=\mu_{0} H_{1},
$$

we deduce from the equations (7), that

$$
B^{\prime}=\frac{\mu_{0} \eta \hat{\xi}}{l \lambda_{x} \lambda_{y} \lambda}\left\{\lambda_{x}^{2}, \lambda_{y}^{2}, \lambda_{z}^{2}\right\} H^{\prime}
$$


Since

$$
\begin{aligned}
B^{\prime} & =B-\frac{1}{c}\left[k, E^{\prime}\right], \\
H^{\prime} & =H-\frac{1}{c}[v, E],
\end{aligned}
$$

and

this gives the relation connecting the magnetic induction $B$ with the electric and magnetic forces $E$ and $H$.

This equation (9) must hold good in the case of a non-magnetizable medium, i.e., when $B=H$. The equation may then be written in the form

$$
H-\frac{1}{c}\left[k, E^{\prime}\right]=\frac{\mu_{0} \xi_{\eta}}{l \lambda_{x} \lambda_{y} \lambda_{z}}\left\{\lambda_{x}^{2}, \lambda_{y}^{2}, \lambda_{z}^{2}\right\} H^{\prime},
$$

or, since

$$
E^{\prime}=E+\frac{1}{c}[v, H]
$$

$$
\begin{aligned}
H-\frac{1}{c}[k, E]-\frac{1}{c^{2}} v(k, H) & +\frac{H}{c^{2}}(k, v) \\
= & \frac{\mu_{0} \xi \eta}{l \lambda_{x} \lambda_{y} \lambda_{z}}\left\{\lambda_{x}^{2}, \lambda_{y}^{2}, \lambda_{z}^{2}\right\}\left\{H-\frac{1}{c}[v, E]\right\} .
\end{aligned}
$$

On writing out this vector equation in full, we should find that it can be satisfied by putting

$$
\begin{gathered}
\mu_{0}=1, \quad k_{x}=\frac{\dot{\xi} \eta}{l \lambda x} \bar{v}=\bar{v}\left(1-\frac{\bar{v}^{2}}{c^{2}}\right)^{-1}=\bar{v} \epsilon, \\
\lambda_{y}=\lambda_{z}=\lambda_{x} \mathrm{e}^{\mathrm{b}} .
\end{gathered}
$$

The various formulø connecting the constants of the transformation can now be brought together into the scheme

$$
\begin{gathered}
k_{x}=\bar{v} \epsilon, \quad k_{y}=0, \quad k_{x}=0, \\
\lambda_{y}=\lambda_{z}=\lambda_{x} \epsilon^{\frac{1}{2}}, \quad \frac{\xi \eta}{l \lambda_{x}}=\frac{\xi}{l \eta^{2} \lambda_{x}}=\epsilon,
\end{gathered}
$$

and

$$
\xi^{2} l=\lambda_{x} \lambda_{y} \lambda_{3} \text {. }
$$

It follows then that $\eta=1$, whence, expressing everything in terms of $\epsilon$ and $\epsilon^{\frac{1}{b}} l(\equiv j)$, we have

$$
k_{x}=\bar{\imath} \epsilon, \quad \lambda_{x}=\epsilon^{-\frac{1}{2} j^{3}}, \quad \lambda_{y}=\lambda_{z}=j^{3}, \quad l=j \epsilon^{-\frac{k}{3}}, \quad \text { and } \xi=j^{4} .
$$

Thus the transformation is fully determined except as to the constant $j$.

\section{Final Scheme of the Transformation.}

In Lorentz's paper in 1904, where the case of a non-magnetizable dielectric is considered, this same indeterminateness occurs, and it is 
there shown that, in order to get consistent results for the longitudinal electromagnetic mass, the value of the constant $j$ must be taken to be unity.

The transformation is thus finally expressible in the following form:-

The equations referred to axes moving with the matter along the direction of the axis of $x^{\prime}$ can be reduced to the form for axes at rest, by the transformation

$$
\begin{gathered}
t_{1}=\epsilon^{-\frac{1}{2}},\left\{t^{\prime}-\frac{\epsilon}{c^{2}} \bar{v} x^{\prime} !, \quad x_{1}=\epsilon^{\frac{1}{2}} x^{\prime}, \quad y_{1}=y^{\prime}, \quad z_{1}=z^{\prime},\right. \\
H^{\prime}=\left\{H_{1 x}, \epsilon^{-\frac{k}{2}} H_{1 y}, \epsilon^{-\frac{1}{2}} H_{1 z}\right\}, \\
E^{\prime}=\left\{E_{1 x}, \epsilon^{-\frac{1}{2}} E_{1 y}, \epsilon^{-\frac{1}{2}} E_{1 z}\right\}, \\
B-\frac{\epsilon}{c}\left[v, E^{\prime}\right]=\left\{B_{1 x}, \epsilon^{\frac{1}{2}} B_{1 y}, \epsilon^{\frac{1}{2}} B_{1 z}\right\}, \\
D+\frac{\epsilon}{c}\left[v, H^{\prime}\right]=\left\{D_{1 \dot{x}}, \epsilon^{\frac{1}{4}} D_{1 y}, \epsilon^{\frac{1}{2}} D_{1 z}\right\}, \\
C=\left\{\epsilon^{-\frac{1}{2}} C_{1 x}, C_{1 y}, C_{1 z}\right\} .
\end{gathered}
$$

The equations become

where

$$
\begin{gathered}
\operatorname{curl}_{1} E_{1}=-\frac{1}{c} \frac{\partial B_{1}}{\partial t_{1}} \\
\operatorname{curl}_{1} H_{1}=\frac{1}{c}\left\{C_{1}+\frac{\partial D_{1}}{\partial t_{1}}\right\}, \\
\operatorname{div}_{1} B_{1}=0, \quad \operatorname{div}_{1} D_{1}=\rho_{1}, \\
\rho_{1}=\left\{\rho-\frac{\epsilon \bar{v} C_{x}}{c^{2}}\right\} e^{-\frac{1}{2}}
\end{gathered}
$$

Further, the relations between the electric displacement, the magnetic induction, the conduction current, and the electric and magnetic forces for the case of matter in motion are

$$
\begin{gathered}
D+\frac{\epsilon}{c}\left[v, H^{\prime}\right]=K_{0}\left\{E_{x}^{\prime}, \epsilon E_{\eta}^{\prime}, \epsilon E_{?}^{\prime}\right\}, \\
B-\frac{\epsilon}{c}\left[v, E^{\prime}\right]=\mu_{0}\left\{H_{x}^{\prime}, \epsilon H_{y}^{\prime}, \epsilon H_{z}^{\prime}\right\}, \\
C=\frac{1}{\sigma_{0}}\left\{E_{x}^{\prime} \epsilon^{-\frac{1}{z}}, E_{\eta !}^{\prime} \epsilon^{\frac{1}{2}}, E_{z}^{\prime} \epsilon^{\frac{1}{\xi}}\right\} .
\end{gathered}
$$


Since

$$
E^{\prime}=E+\frac{1}{c}[v, B]
$$

the second of the above relations can be written in the form

$$
\begin{aligned}
& B-\frac{\epsilon}{c}[v, E]-\frac{\epsilon}{c^{2}} v(v, B)+\epsilon \frac{v^{2}}{c^{2}} B=\mu_{0}\left\{H_{x}^{\prime}, \epsilon H_{y}, \epsilon H_{z}^{\prime}\right\}, \\
& \text { or } \quad B-\epsilon\left\{H-H^{\prime}\right\}-\frac{\epsilon}{c^{2}} v(v, B)+\epsilon \frac{v^{2}}{c^{2}} B=\mu_{0}\left\{H_{x}^{\prime}, \epsilon H_{y}^{\prime}, \epsilon H_{z}^{\prime}\right\} .
\end{aligned}
$$

Therefore, writing out in full this vector equation, which, it must be remembered, only holds good for a velocity whose components are $\bar{v}, 0,0$, we have

Since

$$
\left.\begin{array}{rl}
B_{x} & =\mu_{0} H_{x}^{\prime}=\mu_{0} H_{x} \\
B_{y} & =H_{y}+\left(\mu_{0}-1\right) H_{y}^{\prime} \\
B_{z} & =H_{z}+\left(\mu_{0}-1\right) H_{z}^{\prime}
\end{array}\right\} .
$$

this scheme of equations expresses $B$ in terms of $H$ and $E$ for the case of matter in motion.

No modification of the equation of connection between the magnetic induction and the electric and magnetic forces is used by Lorentz for the case of matter in motion, but the above equation is the same as that used by G. T. Walker in his work on Aberration and the Electromagnetic Field.

For the relation connecting $D$ with $H$ and $E$ we have, in a similar way,

or

$$
\begin{aligned}
D+\frac{\epsilon}{c}[v, H]-\frac{\epsilon}{c^{2}} v(v, E) & +\epsilon \frac{v^{2}}{c^{2}} E=K_{0}\left\{E_{x}^{\prime}, \epsilon E_{y}^{\prime}, \epsilon E_{z}^{\prime}\right\}, \\
D+\frac{\epsilon}{c}[r, H-B]-\frac{\epsilon}{c^{2}} v(v, E)+\epsilon \frac{v^{2}}{c^{2}} E & \\
= & K_{0}\left\{E_{x}^{\prime}, \epsilon E_{y}^{\prime}, \epsilon E_{z}^{\prime}\right\}-\frac{\epsilon}{c}[v, B] \\
& =K_{0}\left\{E_{x}^{\prime}, \epsilon E_{y}^{\prime}, \epsilon E_{z}^{\prime}\right\}-\epsilon\left\{E^{\prime}-E\right\} .
\end{aligned}
$$

Writing this vector equation out in full, the components of the vector $v$ being $\bar{v}, 0,0$, we have

$$
\left.\begin{array}{l}
D_{x}=K_{0} E_{x}^{\prime}=K_{0} E_{x} \\
D_{y}=E_{y}+\epsilon\left(K_{0}-1\right) E_{y}^{\prime}-\frac{\epsilon}{c} \bar{v}\left\{B_{z}-H_{z}\right\} \\
D_{z}=E_{z}+\epsilon\left(K_{0}-1\right) E_{z}^{\prime}+\frac{\epsilon}{c} \bar{v}\left\{B_{y}-H_{y}\right\}
\end{array}\right\} .
$$


The equation of connection in this case, as given by Larmor and Lorentz, is

$$
D=E+\left(K_{0}-1\right) E^{\prime},
$$

which differs essentially from the above form.

For the case of a non-magnetizable medium $B=H$, and the two equations of connection agree up to terms of the tirst order in $\bar{v} / c$.

Finally, the relation connecting $C$ with $E$ and $B$ is

$$
C=\frac{1}{\sigma_{0}}\left\{E_{x}^{\prime} \epsilon^{-\frac{1}{2}}, \epsilon^{\frac{1}{2}} E_{y}^{\prime}, \epsilon^{\frac{1}{2}} E_{z}^{\prime}\right\},
$$

which is the same as that ordinarily given, up to terms of the first order in $\bar{v} / c$.

\section{Comparison with other Theories.}

Cohn accepts the equations of the field as given here, and puts forward as the equations of connection*

$$
\begin{aligned}
D & =K_{0} E^{\prime}-\frac{1}{c}\left[\eta, H^{\prime}\right], \\
C & =\frac{1}{\sigma_{0}} E^{\prime}, \\
B & =\mu_{0} H^{\prime}+\frac{1}{c}\left[\eta, E^{\prime}\right] .
\end{aligned}
$$

On comparing these with the equations as given in this paper, it is easily seen that the difference between the two sets of equations is of the second order, owing to the factor $\epsilon$ which enters into our equations.

An objection to the above equations is that for the free ether, i.e., for $B=H$, and $D=E, \mu_{0}$ and $K_{0}$ do not become unity; nor can the equations be satisfied by assuming aelotropy in $\mu_{0}$ and $K_{0}$, as is the case in the equations in $\S 2$, which differ from those of Cohn in the constant $k$, which latter constant we determined there so as to make the equations consistent in the case of the free ether.

The differences between the equations of connection given in this paper and the equations ordinarily given have been pointed out in $\$ 5$. Here it must be remembered that the equations of connection given by Larmor and Lorentz for the case of matter in motion did not pretend to

* Göttinger Nachrichten, 1901, Vol. 1; Ann. der Physik, Vol. 29, 1902. 
be correct up to the second order in $\vec{v} / c$, so that a difference in this order is to be expected.*

Besides differences of the second order, the equations (11) differ from the equations of Larmor and Lorentz by a term of the first order, agreeing with them up to the first order in the case of non-magnetizable bodies.

A system of equations of a somewhat similar type have recently been given by the late $\mathrm{H}$. Minkowski, + a demonstration of which has also been given by Einstein and Laub. $\downarrow$ The method used in these papers seems to be essentially different from that employed here. Einstein and Laub start from the Maxwellian scheme of equations for matter at rest, and then apply to them the equations of transformation which belong to the Lorentz-Einstein theory of relativity, thereby arriving at the equations of electrodynamics for matter in motion.

The equations thus reached differ from the equations of Larmor and Lorentz, not only as regards the equations of connection, but also as regards the equations of the electromagnetic field:

For the sake of comparison, we give here at length Minkowski's equations.

Referred to axes fixed in space, the equations of the field are

$$
\begin{aligned}
& \operatorname{curl} H=\frac{1}{c}\left\{\frac{\partial D}{\partial t}+C+\rho v\right\}, \\
& \operatorname{curl} E=\frac{1}{c} \frac{\partial B}{\partial t}, \\
& \operatorname{div} D=\rho, \quad \operatorname{div} B=0,
\end{aligned}
$$

whilst the equations of connection are

$$
\begin{gathered}
D+\frac{1}{c}[v, H]=K_{0}\left\{E+\frac{1}{c}[v, B]\right\}, \\
B-\frac{1}{c}[v, E]=\mu_{0}\left\{H-\frac{1}{c}[v, D]\right\}, \\
C=\frac{1}{\sigma_{0}}\left\{E_{x}^{\prime} \epsilon^{-\frac{1}{2}}, E_{y}^{\prime} \epsilon^{\frac{1}{2}}, E_{z}^{\prime} \epsilon^{\frac{\xi}{3}}\right\} .
\end{gathered}
$$

Comparing the equations of the field with those given at the beginning of $\$ 1$, the difference lies in the first equation, where the term curl $[P, v]$ is included in the first equation in $\S 1$. As regards the equations of con-

* Cp. e.g., Larmor, Aether and Matter, p. 113.

† Göttinger Nachrichten, 1908.

$\ddagger$ Annalen der Physik, 1908, Vol. 26, p. 552. 
nection, the first two of Minkowski's equations differ from those given in this paper.

The differences seem to lie in the fact that a non-magnetizable medium is defined by Larmol and Lorentz as a medium in which $B=H$, which condition has been used in $\$ 4$, in finding the constants of the general transformation; according to Minkowski, however, non-magnetizable media are defined by $\mu_{0}=1$, so that for such a medium $B$ is a subsidiary vector defined as equal to

$$
H-\frac{1}{c}[v, D-E]
$$

whence $B$ is no longer equal to $H$, unless at the same time $D=E$, i.e., except in the case of the free ether.

'The first two of Minkowski's equations of connection are, in fact, two simultaneous equations giving $B$ and $D$ in terms of $E$ and $H$.

Solving them, we find*

$$
\begin{aligned}
& D+\frac{K_{0} \mu_{0}}{c^{2}}[v,[v, D]]=K_{0} \nmid E+\frac{1}{c^{2}} \cdot[v,[v, E]] ;+\frac{\left(K_{0} \mu_{0}-1\right)}{c}[v, H], \\
& B+\frac{K_{0} \mu_{0}}{c^{2}}[v,[v, D]]=\mu_{0} \nmid H+\frac{1}{c^{2}}[v,[v, H]]-\frac{\left(K_{0} \mu_{0}-1\right)}{c}[v, E] .
\end{aligned}
$$

Taking as a particular case a direction $z$ perpendicular to the velocity $\bar{v}$, these equations read

$$
\begin{aligned}
& D_{z}\left\{1-K_{0} \mu_{0} \frac{\bar{v}^{2}}{c^{2}}\right\}=K_{0}\left(1-\frac{\bar{v}^{2}}{c^{2}}\right) E_{z}+\frac{\bar{v}}{c}\left(K_{0} \mu_{0}-1\right) H_{y,} \\
& B_{z}\left\{1-K_{0} \mu_{0} \frac{\bar{v}^{2}}{c^{2}}\right\}=\mu_{0}\left(1-\frac{\bar{v}^{2}}{c^{2}}\right) H_{z}-\frac{\bar{v}}{c}\left(K_{0} \mu_{0}-1\right) E_{y} .
\end{aligned}
$$

Comparing these with the corresponding equations as given in $\S 5$, which may be written

$$
\begin{aligned}
& D_{z}=E_{z}\left\{1+\epsilon\left(K_{0}-1\right)+\frac{\epsilon \bar{v}^{2}}{c^{2}} K_{0}\left(\mu_{0}-1\right)\right\}+\frac{\epsilon \bar{v}}{c} H_{y}\left(K_{0} \mu_{0}-1\right), \\
& B_{z}=\mu_{0} H_{z}-\frac{\bar{v}}{c}\left(\mu_{0}-1\right) E_{y},
\end{aligned}
$$

the differences are at once apparent. 
Since

$$
\epsilon=\left(1-\frac{\bar{v}^{2}}{c^{2}}\right)^{-1},
$$

we see that the equations for $D_{z}$ are the same to the first order, viz.,

$$
D_{z}=K_{0} E_{z}+\frac{\bar{v}}{c} H_{\eta}\left(K_{0} \mu_{0}-1\right) .
$$

The equations for $B_{z}$ are, however, different, even up to this order, being respectively

$$
B_{z}=\mu_{0} H_{z}-\frac{\bar{v}}{c}\left(K_{0} \mu_{0}-1\right) E_{y},
$$

and

$$
B_{z}=\mu_{0} H_{\tilde{z}}-\frac{\bar{v}}{c}\left(\mu_{0}-1\right) E_{y} .
$$

This difference, of course, depends on the different conceptions of magnetic media, as stated above, since, according to Minkowski, a non-magnetic medium possesses a magnetic induction $B$, which is not equal to the magnetic force $H$, differing therefrom by a quantity of the first order in $\bar{v} / c$. 